\title{
Acupuncture as a bioinformatics science and a proposal for the recognition of a new law of nature "Law of therapeutic neuromodulation"
}

\author{
Andrés A. Zuno-Arce ${ }^{1}$, Carlos U. Haubi-Segura² \\ ${ }^{1}$ Universidad Candegabe de Homeopatía, Buenos Aires, Argentina \\ ${ }^{2}$ Departamento de Disciplinas Pecuarias, Universidad Autónoma de Aguascalientes, Aguascalientes, México \\ Email: andreszuno@gmail.com
}

Received 1 February 2012; revised 24 April 2012; accepted 29 August 2012

\begin{abstract}
Brain, an organ similar to a computer, is the ultimate director of the body ${ }^{1}$. This means that organs, tissues and cells are under its control and that these parts of the body do not control what happen to them. If the brain is functioning properly, we have health; and if it works wrongly a disease will show. Acting on the brain by neuromodulation, by means of acupuncture, homeopathy or even allopathic drugs can produce a therapeutic response. The purpose of this paper is to recognize this effect and propose a new Law of Cure which explains how several complementary and alternative medicine (CAM) systems work. In this theory, acupuncture works by stimulating peripheral sensory receptors and transmitting information to the brain which in turn will trigger a curative response to the affected organ. In this sense, acupuncture is an informatics system for a biological system, and therefore it could be considered part of the bioinformatics sciences, in the same idea that the brain is a living computer which has inputs and outputs and controls all process inside the body. In this paper we present the basis for this theory.
\end{abstract}

Keywords: Acupuncture; Bioinformatics; Central Nervous System; Computer; Brain; Therapeutic;

Neuromodulation; Law of Nature

\section{INTRODUCTION}

\subsection{A Brief Introduction to Science and the Laws of Nature}

Science is a systematic enterprise that builds and organ-

${ }^{1}$ http://www.nlm.nih.gov/medlineplus/ency/article/002311.htm

The central nervous system is composed of the brain and spinal cord. Your brain and spinal cord serve as the main "processing center" for the entire nervous system, and control all the workings of your body. izes knowledge in the form of testable explanations and predictions about the natural world. Science also is about synthesis, confronting thesis and antithesis in a dialectic fashion, creating new theories based on apparently confronting ideas. Eventually, some theories are elevated to the status of laws of nature, as they become a theoretical principle deduced from particular facts, applicable to a defined group or class of phenomena, and expressible by the statement that a particular phenomenon always occurs if certain conditions be present [1]. In physics, chemistry and mathematics we have several laws (i.e. laws of motion, of electromagnetism and gravitation, the laws of energy (thermodynamics) and heat transfer, the gas laws and the laws of aerodynamics [2]). There are several Laws that apply to physiology, for example, Law of facilitation, Arndt-Schultz Law, Davis' Law, Reciprocal Inhibition, All or Non, Weber's Law, Pfluger's Law, etc. However, in medicine, very few Laws of Cure have been proposed. On the other hand, complementary and alternative medicine (CAM) has a series of laws that direct the actions of the physician, both during diagnosis and treatment. In homeopathic medicine the Law of Similars (similia similibus curentur), proposed by Samuel Hahnemann in 1810, states that any substance that can produce a disease in a healthy individual will cure a person presenting the same symptoms when given the same remedy in a diluted concentration [3]. Another Law of Cure, about the progression of disease and of the healing processes, was presented by Constantine Hering in 1833 [4], which states that: 1) symptoms will reverse in chronological order to how symptoms originally appeared, 2) symptoms will reverse from above to below and from the inside to the outside, and 3) from the most important to the least important organ. This law is also used by acupuncturists, which detect the progression of the healing process accompanied by a therapeutic aggravation, which can be roughly simplified as "it will get worse before it gets better" [5]. 


\subsection{Acupuncture and Traditional Chinese Medicine}

Acupuncture is an alternative medicine system originated in ancient China that treats patients by manipulating thin solid needles which are inserted into acupuncture points in the skin (acupoints). According to Traditional Chinese Medicine (TCM), the stimulation of these points can correct imbalances in the flow of energy (qi) through specific channels (meridians) [6]. In TCM, disease is perceived as a disharmony (or imbalance) in the functions and interactions of yin and yang and a reduction in the qi [7]. Diagnosis is based on inspection, auscultation and olfaction, inquiring, palpation and examination of tongue and pulse [8]. Acupunctural therapy is generally complemented by other therapies, such as moxibustion, which is the use of moxa, or mugwort herb (Artemisia vulgaris L.), to stimulate specific acupoints [9].

The insertion of the needles is performed rapidly through the superficial layers of the skin with very little pain, as can be observed during acupunctural treatment of veterinary patients [10]. Stimulation of the acupoints is performed either through variable movement of the needles or by electroacupuncture as well as by injection of drugs, vitamins or herbal extracts [11].

Therapeutic uses of acupuncture which have been approved by the World Health Organization include pain relief, nausea and vomiting, fertility and childbirth although there is insufficient evidence to determine whether acupuncture is beneficial in other conditions [12]. Placebo effect in humans is a common controversy but it should be ruled out in veterinary acupuncture, as systematic review of clinical trial showed no compelling evidence to reject or recommend acupunctural treatment in domestic animals [13].

According to scientific research, no histological or physiological structures correlate to these principles [14], however, it is subject of serious research by governmenttal agencies [15]. Several theories about the mechanisms underlying pain relief are possibly related to an increased release of endorphins, serotonin, nor epinephrine or GABA [16]. For example, Yoshimoto et al. (2006), discovered that acupuncture stimulates the release of serotonin, but not dopamine, in rats [17]. Most of these theories and counter-theories are based on the premise that cure is produced at a local level, and only very lately, new theories about the role of the Central Nervous System (CNS), and specifically about the neuromodulation of disease, have emerged, creating several societies for its study [18]. Previous publications of the author describe the CNS as a cybernetic, self-regulatory system, in charge of both controlling health and disease, has raised interest and expectations in the medical and scientific community [19].
The informatics-cybernetic conceptualization applied to medicine, synthesized in this document, is a simplification of the understanding of what health, disease, and cure are from a purely physiological point of view. In this context, for example, "disease is a CNS dysfunction" but then, it should be also true that "health is a CNS function”.

\subsection{Therapeutic Bioinformatics and Neuromodulation}

Although bioinformatics is generally regarded as the use of computer science to facilitate research in molecular biology, a broader view of Bioinformatics should include the use of information and systems theory to understand health and disease. Of special interest is the input of sensory stimuli, data processing and output of biological responses coordinated by the CNS, an organ similar to a computer.

Acupuncture, in making the CNS react with its various methods and media, uses the "acupoints" in a way that resembles the way we handle information and correlated media in the mechanical-electronic-brain computers with the keyboard or by means of tactile media, like "touching the screen" or "clicking in the mouse". Acupuncture uses insertion of needles, digital pressure and energies (photonic energy, LASER and heat) to produce an input of information, as one does in computers. When we stimulate a specific acupoint, a nervous impulse is transmitted towards the CNS, which receives the impulse, processes it and reacts accordingly. According to the type of disease treated, either due to a reduction in its metabolism or to a degenerative disease or an increase in the metabolic activity of an organ (inflammation, neoplasia), two basic types of reactions can be expected. Stimulation of a group of acupoints can in turn stimulate the CNS to trigger a positive feedback mechanism that will reactivate the organ, or, on the other hand, another group of acupoints will produce a negative feedback mechanism, regulating the excessive reactivity of an organ or system. In both cases, the process of health was controlled by the CNS and was not a local, isolated, event.

Inflammation is part of a complex biological response of vascular tissues to harmful stimuli, pathogens, damaged cells, or irritants. Although it is correctly regarded as part of the healing process, orthodox medical treatment is generally focused on suppressing it with the use of non-steroidal anti-inflammatory drugs (NSIADs) and steroids (cortisol and its derivates). By acting on the organism as a whole, and not only locally, acupuncture, homeopathy and herbal treatments, are used to control, rather than to inhibit, the inflammatory response by stimulating it via the CNS, through neuromodulation.

In understanding acupuncture in this way, we may call 
it bioinformatics. Also we see that each and every health science that is based on the handing of the cybernetics-informatics systems of the living organisms are bioinformatics. For example, psychotherapy, which tries to modify the workings of the mind (a CNS function) through reprocessing of a persons thoughts, also uses the neuromodulation capacity of the organism, producing changes in conduct and psychosomatic manifestations. Even chemical drugs used as a support for psychiatric therapy use neuromodulation as a mechanism of action.

Even the placebo effect is another neuromodulation healing process, where the patient takes a pill believing it has a real therapeutic effect (input), processes the information in the CNS, and produces and healing or therapeutic effect (output).

\section{DISCUSSION}

\subsection{The CNS Is Responsible for Healh}

There is a common consensus in the scientific community that the CNS is responsible for running the organism as a whole. This means that health, keeping the economy properly functioning, is its obligation and that is what it does. If we look at this from another point of view, we understand that the organs are not responsible for their proper functioning, their health, because, as we just saw, such responsibility is the brain's. Thus, neither the heart nor the liver or any other organs are the architects of their own destiny. It is the brain. The same happens with tissues and cells. For example, the elements that require chemical energy for muscle function, synthesized by the liver, are carried by the circulatory system, which is the responsibility of the CNS. And for the liver to function correctly it needs a number of variables that are only controlled by the brain and in no way by de liver itself. Call any other organ and it will be the same picture.

\subsection{The CNS Is Responsible for Sickness}

The conclusion of this series of events is that the organs are not either responsible for their disease and that it is the brain's.

This concept does not apply in cases in which the body is affected by external agents such as toxins, or energies like electricity or heat.

So, disease is a reflex of a dysfunction of the brain.

\subsection{The CNS Is Responsible for the Healing Process}

In this context we can suggest that the healing process is also the responsibility of the CNS and not an independent process of each organ, tissue or cell. Most notably the cell, by virtue of her staple constituent of tissues and organs, do not have any control command. In fact, cells can be regarded as babies who need to be provided of everything they need and eliminate what is harmful to them, and this is an exclusive task of the CNS, and that is what acupuncture does.

In a recent study, Prahlad and Morimoto (2011) found that neuronal cells of $C$. elegans respond differently to acute stress such as heat shock and chronic stress caused by the expression of misfolded proteins, suggesting that neuronal signaling determines the course of cellular proteotoxicity [20].

\subsection{Thesis, Antithesis and Synthesis}

As an antithesis to previous medical paradigm, where the CNS controls only homeostasis but not disease, our personal point of view is that the CNS also controls disease. Synthesis of both theories suggests that the CNS is responsible for health, disease and cure. Acting on the brain acupuncture can heal organs, tissues and cells, which is definitely proven in clinical research and this is precisely the mechanism by which this therapy works. This new theory provides a new medical and physiological paradigm: acupuncture (and other medical therapies) actually works through neuromodulation.

\subsection{Acupuncture Proved by Neuromodulation}

Neuromodulation by acupuncture has been proven through experimentation using imaging technologies, such as fMRI, PET and SPECT. Hui et al. (2000) evaluated how acupuncture modulates the limbic system and subcortical gray structures of the human brain using fMRI studies in normal subjects [21]. Lewith et al. (2005) reviewed research involving acupuncture using brain imaging techniques in order to show the specific areas of the brain influenced when acupuncture was used [22].

The current state of knowledge regarding the effect of acupunctural therapy on humans and animals suggests that specific areas of brain are influence when acupuncture is used. Additional papers on acupuncture neuromodulation: can be found at several websites (i.e. http:// www.equimagenes.com/index.php/resonancia-pet-acupu ntura). Dhond et al. (2007) reviewed several neuroimaging techniques at the moment where acupuncture treatment was performed [23]. For example, different central manifestations were found if electroacupuncture was applied at analgesic and no analgesic acupoint in rats [24]. Also, Yan et al. (2005) found specific fMRI patterns in human brain triggered by stimulation of special acupoints [25]. Woo et al. (2006) demonstrated in doubleblind trials to stimulate or inhibit brain function by the use electroacupuncture [26]. Similar results were obtained by Kong et al. (2002), however different brain networks were activated when using manual or electroacupuncture stimulation of acupoints in normal subjects [27]. 
All data points at the possibility that acupuncture works by stimulating the CNS and then it is up to the brain and other CNS structures to produce a healing process.

\section{CONCLUSION}

Acupuncture is, in a sense, a bioinformatics science; with physiological signal processing (needles sending signals to the CNS) and the brain acting like a biological computer and producing a specific output. Cybernetic and self-regulatory mechanisms (homeostasis and homeorresis) help maintain the internal milieu and restore the health status of the patient. This new view offers a novel application tools in medicine and physiology: understanding how the organisms heals itself. Acupuncture accomplishes its healing processes through an effect on the CNS, and therefore it is important to recognize that it follows a Law of Cure: "Law of Therapeutic Neuromodulation".

\section{REFERENCES}

[1] Wikipedia Contributors (2012) Science. http://en.wikipedia.org/wiki/Science

[2] Wikipedia Contributors (2012) Laws of nature. http://en.wikipedia.org/wiki/Laws_of_Nature

[3] Hahnemann, S. (1810) Organon of the medical art. http://www.homeopathyhome.com/reference/organon/org anon.html

[4] Vithoulkas, G. (1980) The science of homeopathy. Grove Press, New York.

[5] Juliano, L. (1995) Laws of cure: Getting worse before getting better.

http://www.reikistudio.com/law_of_cure.pdf

[6] Maciocia, G. (1989) The foundations of Chinese medicine: A comprehensive text for acupunturists and herbalists. 2nd Edition, Churchil Livingston, Edinburgh.

[7] Kaptchuk, T.J. (2000) The web that has no weaver: Understanding Chinese medicine. Contemporary Books, McGraw-Hill, New York.

[8] Tietao, D. (2004) Practical diagnosis in traditional Chinese medicine. Translated by Ergil, M. and Sumei, Y., Churchil Livingston, Edinburgh.

[9] Xin-Nong, C. (2010) Chinese acupuncture and moxibustion. 3rd Edition, Foreign Languages Press, Beijing.

[10] Xie, H. and Preast, V. (2007) Xie's veterinary acupuncture. Blackwell Publishing Professional, Ames. doi:10.1002/9780470344569

[11] Aung, S.K.H. and Chen, W.P.D. (2006) Clinical introduction to medical acupuncture. Thieme Medical Publishers, New York.

[12] WHO (1997) Acupuncture review and analysis of reprots on controlled clinical trials. WHO Consultation on Acupuncture, World Health Organization. http://apps.who.int/medicinedocs/pdf/s4926e/s4926e.pdf
[13] Habacher, G., Pittler, M.H. and Ernst, E. (2008) Effectiveness of acupuncture in veterinary medicine: Systematic review. Journal of Veterinary Internal Medicine, 20, 480-488.

http://onlinelibrary.wiley.com/doi/10.1111/j.1939-1676.2 006.tb02885.x/pdf doi:10.1111/j.1939-1676.2006.tb02885.x

[14] Singh, S. and Ernest, E. (2008) Trick or treatment: The undeniable facts about alternative medicine. Bantam Books, New York.

[15] National Institutes of Health (1997) Acupuncture. NIH Consensus Statement, 3-5 November 1997, 15, 1-34.

[16] Omura, Y. (1989) Connections found between each meridian (heart, stomach, triple burner, etc.) \& organ representation area of corresponding internal organs in each side of the cerebral cortex; release of common neurotransmitters and hormones unique to each meridian and corresponding acupuncture point \& internal organ after acupuncture, electrical stimulation, mechanical stimulation (including shiatsu), soft laser stimulation or QI Gong. Acupuncture \& Electrotherapeutics Research, 14, 155186.

[17] Yoshimoto, K., Fukuda, F., Hori, M., Kato, B., Kato, H., Hattori, H., Tokuda, N., Kuriyama, K., Yano, T. and Yasuhara, M. (2006) Acupuncture stimulates the release of serotonin, but not dopamine, in the rat nucleus accumbens. Tohoku Journal of Experimental Medicine, 208 321-326. doi:10.1620/tjem.208.321

[18] International Neuromodulation Society (2012) Which has an indexed journal: Neuromodulation. Technology at the Neural Interface. http://www.neuromodulation.com

[19] Zuno Arce, A.A. (2005) Homeopatía e Informática: Bioinformática: Manejo curativo de la computadora-cerebro. Ed. Andrés Amado Zuno Arce, Guadalajara. http://www.librosdehomeopatia.es

[20] Prahlad, V. and Morimoto, R.I. (2011) Neuronal circuitry regulates the response of Caenorhabditis elegans to misfolded proteins.

http://www.pnas.org/content/early/2011/08/10/11065571 08.abstract.

[21] Hui, K.K., Liu, J., Makris, N., Gollub, R.L., Chen, A.J., Moore, C.I., Kennedy, D.N., Rosen, B.R. and Kwong, K.K. (2000) Acupuncture modulates the limbic system and subcortical gray structures of the human brain: Evidence from fMRI studies in normal subjects. Human Brain Mapping, 9, 13-25. doi:10.1002/(SICI)1097-0193(2000)9:1<13::AID-HBM2 $>3.0 . \mathrm{CO} ; 2-\mathrm{F}$

[22] Lewith, G.T., White, P.J. and Pariente, J. (2005) Investigating acupuncture using brain imaging techniques: The current state of play. eCAM, 2, 315-319.

[23] Dhond, R.P., Kettner, N. and Napadow, V. (2007) Neuroimaging acupuncture effects in the human brain. Journal of Alternative and Complementary Medicine, 6, 603-616. doi:10.1089/acm.2007.7040

[24] Chiu, J.H., Chung, M.S., Cheng, H.C., Yeh, T.C., Hsieh, J.C., Chang, C.Y., Kuo, W.Y., Cheng, H. and Ho, L.T. (2003) Different central manifestations in response to electroacupuncture at analgesic and nonanalgesic acupoints in 
rats: A manganese-enhanced functional magnetic resonance imaging study. Canadian Journal of Veterinary Research, 2, 94-101.

[25] Yan, B., Li, K., Xu, J., Wang, W., Li, K., Liu, H., Shan, B. and Tang, X. (2005) Acupoint-specific fMRI patterns in human brain. Neuroscience Letters, 383, 236-240. doi:10.1016/j.neulet.2005.04.021

[26] Woo, Y.M., Lee, M.S., Nam, Y., Cho, H.J. and Shin, B.C. (2006) Effects of contralateral electroacupuncture on brain function: A double-blind, randomized, pilot cli- nical trial. Journal of Alternative and Complementary Medicine, 12, 813-815. doi:10.1089/acm.2006.12.813

[27] Kong, J., Ma, L., Gollub, R.L., Wei, J., Yang, X., Li, D., Weng, X., Jia, F., Wang, C., Li, F., Li, R. and Zhuang, D. (2002) A pilot study of functional magnetic resonance imaging of the brain during manual and electroacupuncture stimulation of acupuncture point (LI-4 Hegu) in normal subjects reveals differential brain activation between methods. Journal of Alternative and Complementary Medicine, 8, 399-401. 